\title{
Relación entre el tipo de ejercicio físico y la fatiga cuantificada mediante VFC, CK y el lactato en sangre \\ Relationship between physical exercise type and fatigue quantified through HRV, CK, and blood lactate
}

\author{
*Germán Hernández-Cruz, **Edson Francisco Estrada-Meneses, **Arnulfo Ramos-Jiménez, *Blanca Rocío Rangel- \\ Colmenero, ***Luis Felipe Reynoso-Sánchez, *Janeth Miranda-Mendoza, **José Trinidad Quezada-Chacón \\ *Universidad Autónoma de Nuevo León (México), **Universidad Autónoma de Ciudad Juárez (México), ***Universidad Autónoma de \\ Occidente (México)
}

\begin{abstract}
Resumen: Los atletas son expuestos a cargas de alta intensidad en la búsqueda del rendimiento deportivo, sin embargo, sus efectos no se evalúan apropiadamente. El presente estudio analiza los efectos de cuatro diferentes tipos de ejercicios sobre la variabilidad de la frecuencia cardíaca (VFC), diagrama de Poincaré (SD1: desviación estándar 1 y SD2: desviación estándar 2), así como la Creatina Cinasa (CK) y las concentraciones de lactato en sangre como marcadores de fatiga. Para lograr el objetivo, participaron 10 voluntarios sanos quienes se expusieron a fatiga mediante protocolos de ejercicio isotónicos, isométricos, aeróbicos y anaeróbicos. Se tomaron muestras de la SD1 y de la SD2 para posteriormente probar el comportamiento de la fatiga mediante el índice de estrés (SS) como parámetro simpático, y el índice simpático/parasimpático (S/PS), además se midió la raíz cuadrada media de las diferencias sucesivas (rMSSD) como indicadores parasimpáticos. Se recolectaron muestras sanguíneas al comienzo y al final de cada uno de los tipos de ejercicio para determinar los niveles de CK y lactato. La SD1 disminuye en cada protocolo de ejercicio, mientras que el SS y el S/PS incrementan. Lactato y CK incrementan al final de cada protocolo y correlacionan positivamente con el SD1 y S/PS. LaVFC, CK y lactato son marcadores sensibles para la detección de fatiga, son sensibles tanto al tipo, duración e intensidad del ejercicio, siendo laVFC un marcador no invasivo y novedoso, simple y útil para los entrenadores y atletas. Palabras clave: Rendimiento deportivo, biomarcadores de estrés físico, pruebas de ejercicio físico.
\end{abstract}

\begin{abstract}
Athletes are exposed to high-intensity loads to promote athletic performance, however, the effects are not evaluated appropriately. This study investigates the effects of four types of exhaustion exercises on Heart RateVariability (HRV) and Poincaré features (SD1: Standard deviation 1and SD2: Standard deviation 2), Creatine Kinase (CK) and blood lactate concentrations as biomarkers of fatigue. To achieve this purpose, 10 healthy volunteers were exposed to exhaustive exercise using isotonic, isometric, aerobic and anaerobic fatigue protocols. HRV Poincaré features, standard deviation of instantaneous beat-to-beat R-R interval variability (SD1) and standard deviation of continuous longterm R-R interval variability (SD2) variables were collected. Fatigue was tested through the sympathetic stress index (SS), the index sympathetic/parasympathetic index (S/PS) and the root Mean Square of the Successive Differences (rMSSD) as parasympathetic index. Blood samples were collected at the beginning and at the end of the exercises to determine CK and lactate. The SD1 decreased in each exercise protocol, while the SS and S/PS increased. Lactate and CK increased at the end of each protocol and correlated with SD1 and S/PS. HRV, $\mathrm{CK}$, and lactate are acute markers to detect fatigue, are sensitive to the type, duration, and intensity of exercise, being HRV a novel noninvasive marker, simple and useful for sports coaches and athletes.
\end{abstract}

Keywords: Sports performance, physical stress biomarkers, physical performance test.

\section{Introducción}

Los deportistas son expuestos a cargas de trabajo sistemáticas y de alta intensidad durante los entrenamientos para mejorar el rendimiento deportivo, es por ello, que los entrenadores continuamente implementan métodos para el control de las cargas de trabajo y monitoreo de las adaptaciones en respuesta al entrenamiento, así como, para reducir lesiones y evitar el sobreentrenamiento (Buchheit, Voss, Nybo, Mohr \& Racinais, 2011). Sin embargo, la mayoría de estos procesos de control se aplican sin conocer su adecuada validez y confiabilidad, incapacitándonos el probar los efec-

Fecha recepción: 14-05-21. Fecha de aceptación: 19-10-21

José Trinidad Quezada Chacón

jose.quezada@uacj.mx tos reales de los programas en el desarrollo atlético y la fatiga, y suelen apoyar sus observaciones en la experiencia empírica (Erickson, Côté \& Fraser-Thomas, 2007; Halson, 2014). Lo anterior señala la importancia de la aplicación del método científico en estos procesos, y comprender, si las cargas de ejercicio son aplicadas efectivamente y asimiladas de forma adecuada por el atleta, evitando la fatiga excesiva y progresivamente el sobreentrenamiento.

La fatiga se considerada como la incapacidad momentánea para generar fuerza (Grassi, Rossiter \& Zoladz, 2015), producto de la sucesiva aplicación de cargas de entrenamiento acumuladas y esta ha sido vinculada y necesaria como parte del proceso de entrenamiento, sin embargo, un control inadecuado de las cargas y la relación fatiga-descanso puede provocar con el tiempo una sobrecarga no funcional llamada síndrome de fatiga 
crónica, que se relaciona con la disminución del rendimiento y el sobreentrenamiento (Halson \& Jeukendrup, 2004), esto hace resaltar la importancia de llevar a cabo la monitorización del balance carga-recuperación, existiendo la necesidad de implementar evaluaciones físicas y psicológicas periódicas, confiables y prácticas de los efectos de la fatiga en el ámbito deportivo (ReynosoSánchez et al., 2016).

Hoy en día, existen diversos marcadores fisiológicos y bioquímicos que nos brindan información confiable de la fatiga en dependencia del tiempo que transcurre desde la aplicación de la carga de trabajo y la evaluación, entre ellos están la variabilidad de la frecuencia cardiaca (VFC), concentraciones sanguíneas de Creatina Cinasa (CK) y lactato (Nosaka \& Clarkson, 1996; Ramos-Jiménez, Hernández-Torres, Wall-Medrano, Juarez-Oropeza \& Vera-Elizalde, 2013; Vernillo et al., 2015). En la actualidad la VFC es una herramienta novedosa y no invasiva para medir la actividad simpática y parasimpática del sistema nervioso autónomo (SNA) sensible a las cargas de trabajo (Naranjo, de la Cruz, Sarabia, de Hoyo \& Domínguez, 2015; De la Cruz, López \& Naranjo, 2008).

La literatura reporta un gran número de indicadores de la VFC para evaluar el balance autonómico, incluyendo tanto los análisis de tipo lineal como los no lineales (Zulfiqar, Jurivich, Gao \& Singer, 2010), dominio de frecuencia (Lucini et al., 2017), diagrama de Poincaré (Medina, de la Cruz, Garrido, Garrido \& Naranjo, 2012) sin embargo, hoy en día los marcadores más utilizados en deportistas son la rMSSD y recientemente los índices: estrés score (SS) y el ratio simpático-parasimpático nombrado S/PS Ratio mostrando alta sensibilidad al balance autonómico (Naranjo et al., 2015).

En la práctica deportiva, se ha analizado la fatiga periférica mediante marcadores biológicos como la CK y el lactato. La CK es una enzima intracelular comúnmente empleada para determinar el daño en el tejido cardíaco y esquelético, incrementando su presencia en la sangre tras ejercicio intenso (Ascensao, Rebelo, Oliveira, Marques, Pereira \& Magalhaes, 2008; Nosaka \& Clarkson, 1996). Por otro lado, el lactato incrementa a medida que aumenta la glucólisis anaeróbica y se relaciona exponencialmente con el incremento de la intensidad del ejercicio (Gladden, 2004).

Como se ha mencionado laVFC, CK y lactato se han estudiado mayormente de manera aislada para conocer el efecto de las cargas de entrenamiento sobre el organismo de los atletas, así como el estado de recuperación tras diferentes intensidades de carga (Ascensao, Rebe- lo, Oliveira, Marques, Pereira \& Magalhaes, 2008; Naranjo et al. 2015; Ramos Jiménez et al., 2013). Sin embargo, no se han estudiado estos tres marcadores de manera simultánea, ni cómo se relacionan entre sí en diferentes protocolos de ejercicio. Por lo anterior, el objetivo de este estudio fue analizar el efecto de cuatro diferentes protocolos de ejercicios que inducen fatiga, sobre la VFC, la CK y lactato cómo biomarcadores de fatiga.

\section{Metodología}

\section{Participantes}

Diez jugadores de balonmano de nivel universitario participaron de manera voluntaria en el estudio con alcance transversal $(21.1 \pm 1.72$ años, $1.73 \pm 0.05 \mathrm{~m}$ de estatura, $81.1 \pm 16.75 \mathrm{~kg}$ de peso corporal, $26.5 \pm 4.0$ $\mathrm{kg} / \mathrm{m}^{2}$ de índice de masa corporal, $47.8 \pm 6.42 \mathrm{ml} /$ $\mathrm{kg} / \mathrm{min}$ de $\left.\mathrm{VO}_{2} \max \right)$. Los criterios de inclusión fueron: edad $^{3} 18$ años, tener por lo menos 3 años de experiencia deportiva, asistencia a los entrenamientos, se excluyeron aquellos quienes fumaran, consumirán alcohol $(>$ $100 \mathrm{ml} /$ semana) u otro tipo de drogas, estuvieran bajo tratamiento hormonal o alimenticio para ganar o perder peso, tener impedimentos físicos o lesiones que imposibiliten el máximo rendimiento en las pruebas físicas. El protocolo utilizado fue aprobado por el Comité de Bioética de la Universidad Autónoma de Cd. Juárez (CBE-ICB-069-10-15) y se realizó bajo los lineamientos de la declaración de Helsinki.

\section{Diseño experimental}

En la primera sesión se explicó el procedimiento a los participantes y firmaron el consentimiento informado. Se les aplico el Cuestionario General de Salud y el Cuestionario de Preparación para la Actividad Física (PAR Q +) (Bredin, Gledhill, Jamnik \& Warburton, 2013) para conocer su estado de salud. Posteriormente, para conocer el estado de salud se les solicitó responder el Cuestionario General de Salud y el Cuestionario de Preparación para la Actividad Física (PAR Q +) (Bredin, Gledhill, Jamnik \& Warburton, 2013).

En la segunda sesión, los participantes realizaron una prueba de ejercicio incremental (9-15 min). Posteriormente, bajo un diseño por bloques, los participantes se colocaron aleatoriamente en cuatro diferentes pruebas de ejercicio máximo (PEM: sesiones 3-6) con diferente duración: prueba aeróbica (5-9 min), prueba anaeróbica (2-3 $\mathrm{min})$, prueba de resistencia isométrica (0.7-2.3 min) y prueba de resistencia isotónica (2-7 min) (Figura 
1). Para disminuir el efecto de cada tratamiento sobre los resultados, durante estas cuatro últimas sesiones los participantes acudieron al laboratorio cada $72 \mathrm{~h}$ para realizar las PEM. En cada PEM se registraron las concentraciones de HR, HRV, así como lactato y CK. La temperatura del laboratorio se mantuvo entre 23-24 ${ }^{\circ} \mathrm{C}$.

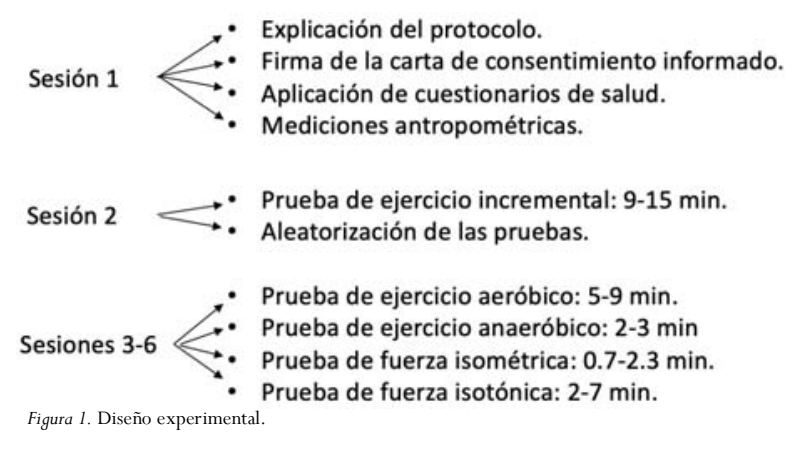

\section{Procedimientos}

Prueba de ejercicio incremental para evaluar el consumo máximo de 02 (VO2max)

Se realizó una prueba de esfuerzo incremental máxima siguiendo los lineamientos del American College of Sport Medicine (ACSM, 2016) para determinar la carga de trabajo máxima y el $\mathrm{VO}_{2} \max$, mediante el uso de una bicicleta ergométrica (Monark ergomedic 828 E, Monark exercise AB, 105 Vansbro, Sweden). La cadencia de la bicicleta se mantuvo a $60 \mathrm{rpm}$. El VO 2 max fue medido mediante un analizador de gases (Cortex MetaLyzer $\mathbb{R}$ 3B, Germany) previamente calibrado el sensor de flujo y las concentraciones de $\mathrm{O}_{2} \mathrm{y} \mathrm{CO}_{2}$, según lo dicta el manual del fabricante (Cortex. Operador's Manual Metalyser 3B, 2016). La prueba fue considerada máxima cuando al menos se presentaron tres de los siguientes parámetros: Fatiga voluntaria (no se puede mantener la cadencia ${ }^{3} 60 \mathrm{rpm}$ por más de 15 segundos) relación del intercambio respiratorio (RIR) mayor a 1.1 ; lactato mayor a $8.0 \mathrm{mmol} / \mathrm{L}$; percepción del esfuerzo ${ }^{3} 8$ (Escala de Borg CR-10); frecuencia cardíaca ${ }^{3}$ 90\% de la FC teórica acorde a la edad; y una meseta en elVO $\max$ (incrementos menores de 150ml/ min a pesar del incremento en la carga de trabajo).

\section{Prueba incremental aeróbica}

La prueba comenzó con una resistencia de $100 \mathrm{~W}$ durante los primeros dos minutos, con incrementos de 25 watts cada minuto, manteniendo una cadencia de 60$70 \mathrm{rpm}$. La prueba finalizó cuando el participante no pudiera mantener una cadencia ${ }^{3} 60 \mathrm{rpm}$ por más de 15 segundos. Para esta prueba se utilizó una bicicleta ergométrica VIAsprint 150 P (Franklin Lakes, NJ, US).

\section{Prueba anaeróbica}

La resistencia aplicada fue constante e igual a la resistencia máxima alcanzada durante la prueba de ejercicio incremental. La cadencia de pedaleo fue de 60-70 rpm. La evaluación finalizó cuando el participante no pudiera mantener una cadencia ${ }^{3} 60 \mathrm{rpm}$ por más de 15 segundos. Para esta prueba se utilizó una bicicleta ergométrica VIAsprint 150 P (Franklin Lakes, NJ, US).

\section{Prueba isotónica}

Los participantes se colocaron en posición sentado con su pierna dominante junto al equipo para medir la fuerza muscular (Primus RS, Maryland, US), el dinamómetro del equipo se colocó a la altura de la rodilla. Previo a la prueba isotónica o isométrica, se ejecutó una contracción máxima voluntaria (CMV) del cuádriceps durante 5 segundos en un ángulo de $45^{\circ}$, estableciendo así el máximo pico de CMV durante el procedimiento de la prueba. Posteriormente, se programó el equipo a un 30\% del CMV registrado previamente, asegurando así que el participante pudiera llevar a cabo la repetición de las contracciones hasta la fatiga, con una frecuencia de $30 \mathrm{rpm}$, registrada mediante un metrónomo digital (Korg MA-1, UK), y con un rango de movimiento de $90^{\circ}$. La prueba finalizaba cuando el participante no pudiera mantener la cadencia ${ }^{3} 30$ rpm por más de 5 segundos.

\section{Prueba isométrica}

Se programó el equipo Primus RS (Maryland, US) al 100\% del CMV. Posteriormente, se pidió al participante realizar una contracción isométrica máxima en un ángulo de $45^{\circ}$. La prueba finalizó cuando el participante no fue capaz de mantener la carga máxima programada por más de 5 segundos.

\section{Antropometría, lactato, CK, FC y VFC}

El peso corporal se registró mediante una báscula electrónica (SECA 656 digital scale, Hamburg, Germany), y la estatura fue medida mediante un estadiómetro de pared (Portable SECA 206 stadiometer, Hamburg, Germany), el sujeto vestía ropa ligera y sin calzado. Para analizar las concentraciones en sangre de lactato, se recolectaron muestras de sangre capilar (25 $\mu \mathrm{L}$ de la yema del dedo) antes e inmediatamente al finalizar cada prueba, se utilizó un tubo de vidrio capilar heparinizado y analizado en el mismo momento por medio de un analizador de lactato (YSI Model 1500,YSI incorporated, Yellow Springs, OH). El análisis de concentraciones de CK se llevó a cabo mediante la toma de 
muestras de sangre capilar (500 $\mu \mathrm{L}$ de la yema del dedo) previo al ejercicio, así como 10 minutos posterior a completada cada prueba, analizados de manera inmediata utilizando el equipo Reflotron (Boehringer Mannheim GmbH, Mannhein, Germany).

La frecuencia cardiaca (FC) y VFC fueron tomadas en tres momentos mediante un dispositivo de telemetría con corto alcance (Polar Team 2, Kempele, Findland). Las tomas fueron antes, durante y después de las pruebas: 5 minutos para la toma basal (posición supina), durante el tiempo de duración de la prueba, y 5 minutos post recuperación al término de la prueba (posición supina). Para analizar el grado de influencia de la rama parasimpática se utilizó la rMSSD. Además de los índices del diagrama de Poincaré donde: SD1 es un indicador de actividad parasimpática (SNP); mientras que el inverso de SD2 es utilizado para el cálculo del índice de estrés (SS: 1000 x 1/SD2), así como el ratio simpático/parasimpático (S/PS), los cuales son indicadores de actividad simpática y balance autónomo respectivamente (Naranjo et al., 2015). Los datos fueron analizados mediante el software Kubios HRV versión 3.1 (University of Kuopio, Kuopio, Finland) utilizando la corrección automática de artefactos.

\section{Análisis estadístico}

Se utilizó la prueba de Shapiro-Wilk para analizar la normalidad de los datos. Una vez verificada la distribución normal, se emplearon los análisis paramétricos. Para analizar el posible efecto del estado nutricional medido mediante el IMC y la capacidad aeróbica obtenida a través del $\mathrm{VO}_{2}$ max sobre las variables dependientes (SD1, SD2, SS, S/PS, lactato y CK), se empleó un análisis multivariante, en el modelo se incluyó tanto el IMC como el $\mathrm{VO}_{2} \max$ como covariables. Posteriormente, para analizar el efecto del tiempo (línea base, ejercicio y recuperación) entre los diferentes protocolos de ejercicio, se utilizó el modelo lineal general (MLG) de medidas repetidas, siendo el tiempo como la variable intra-sujeto y los protocolos de ejercicio como factores internos. Se evaluaron las diferencias entre los tiempos mediante la prueba de Bonferroni. Adicionalmente, se ejecutó un ANOVA de una-vía para analizar las diferencias entre las pruebas de forma independiente. Cuando las varianzas entre los tratamientos fueron diferentes, las prueba post hoc de T2 de Tamhane y HSD de Tukey fueron aplicadas. Finalmente, se aplicó una prueba de correlaciones bivariadas de Pearson. Los resultados son presentados en las tablas como medias \pm desviación estándar (DE) y el intervalo de confianza al 95\% (IC 95\%) y su tamaño del efecto (parcial $h^{2}$ ). Todos los análisis se realizaron utilizando el paquete estadístico SPSS v21 (SPSS Inc, Chicago, IL).

\section{Resultados}

Como se observa en la tabla 1, la rMSSD tuvo un comportamiento similar entre los protocolos, en cambio, la SD1 en la toma recuperación del protocolo aeróbico fue menor que en el isotónico e isométrico $(p<.05)$. No se presentaron diferencias significativas del SS entre los protocolos en ninguna toma (base, ejercicio y recuperación). El ratio S/PS fue mayor en la toma ejercicio durante el ejercicio anaeróbico en comparación con los protocolos isotónicos e isométricos $(p<.05)$.
Tabla 1

\begin{tabular}{|c|c|c|c|c|c|}
\hline & a)Isotónico & a)Isométrico & a)Anaeróbico & a)Aeróbico & $\eta^{2}$ Parcial \\
\hline \multicolumn{6}{|l|}{ MSSD } \\
\hline asal & $68 \pm 31(52-86)$ & $83 \pm 47(55-110)$ & $110 \pm 96(59-173)^{2,3}$ & $66 \pm 30(51-86)^{2,3}$ & 0.09 \\
\hline jercicio & $19 \pm 27(8-37)^{1,3}$ & $19 \pm 13(11-27)^{1}$ & $18 \pm 42(3-46)$ & $7 \pm 6(4-11)$ & 0.04 \\
\hline ecuperación & $55 \pm 35(36-77)$ & $76 \pm 53(45-107)$ & $25 \pm 45(7-54)$ & $14 \pm 16(7-25)$ & 0.30 \\
\hline asal & $49 \pm 22(33-64)$ & $59 \pm 33(35-83)$ & $78 \pm 68(22-133)^{2,3}$ & $56 \pm 17(43-68)^{2,3}$ & 0.07 \\
\hline jercicio & $13 \pm 19(0-27)^{1}$ & $13 \pm 9(7-20)^{1,3}$ & $13 \pm 30(0-37)$ & $5 \pm 4(2-8)$ & 0.04 \\
\hline ecuperación & $39 \pm 25(21-57)$ & $54 \pm 37(27-81)$ & $18 \pm 32(0-42)$ & $10 \pm 11(2-18)^{\mathrm{a}, \mathrm{b}}$ & 0.30 \\
\hline sal & $10 \pm 3(8-12)$ & $11 \pm 4(8-13)$ & $8 \pm 4(6-11)$ & $8 \pm 2(6-9)$ & 0.13 \\
\hline jercicio & $13 \pm 5(9-16)$ & $11 \pm 6(7-15)$ & $17 \pm 10(11-23)^{1}$ & $12 \pm 8(6-18)$ & 0.10 \\
\hline ecuperación & $13 \pm 5(9-17)$ & $13 \pm 12(4-21)$ & $22 \pm 17(9-35)$ & $22 \pm 11(14-30)^{1}$ & 0.14 \\
\hline asal & $0.3 \pm 0.3(0.1-0.5)^{2}$ & $0.3 \pm 0.4(0.1-0.6)$ & $0.2 \pm 0.2(0.1-0.4)^{2}$ & $0.1 \pm 0.1(0.1-0.2)^{2,3}$ & 0.13 \\
\hline jercicio & $2.3 \pm 1.8(1.1-3.6)$ & $1.6 \pm 1.6(0.4-2.7)$ & $6.9 \pm 5.7(2.8-10.9)^{\mathrm{a}, \mathrm{b}}$ & $4.1 \pm 4.0(1.2-6.9)$ & 0.10 \\
\hline ecuperación & $0.7 \pm 0.9(0.1-1.3)$ & $2.0 \pm 5.3(0-5.7)$ & $6.4 \pm 9.2(0-13.0)$ & $5.2 \pm 4.4(2.0-8.3)$ & 0.14 \\
\hline
\end{tabular}

En el diagrama de Poincaré (SD1 y SD2) disminuye durante el ejercicio, principalmente en su componente parasimpático (SD1), sin lograr una recuperación completa durante los 5 minutos post ejercicio. Además, du-

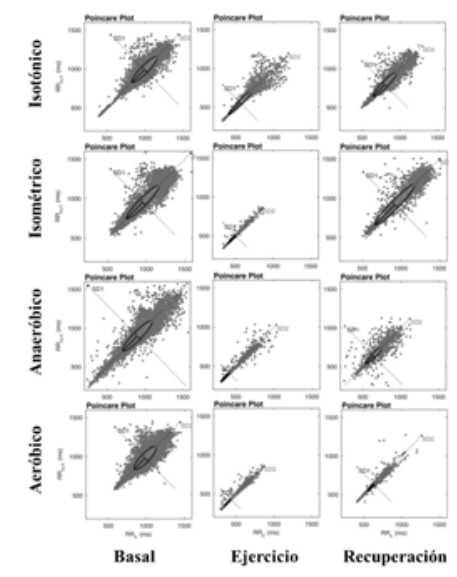

Figura 2. Modelo cualitativo de Poincaré entre las diferentes pruebas y tomas. 
rante la toma recuperación, el componente parasimpático fue menor en el protocolo aeróbico, seguido del anaeróbico, isotónico e isométrico (Figura 2).

La Figura 3 (a, b y c) confirman en parte lo que se observa en la Tabla 1 y la Figura 2, lo cual es la presencia de una recuperación rápida de los niveles basales en el protocolo isotónico, seguido del isométrico con el aeróbico y anaeróbico $(p<.05)$. Mediante el análisis GLM de muestras repetidas, se observaron diferencias entre la toma basal vs. Ejercicio y recuperación en las variables SS y S/PS $(p<.01)$. Para la SD1, se presentaron diferencias entre las tres tomas $(p<.01)$. El análisis multivariante mostró tanto el IMC como elVO max no influyeron significativamente entre los parámetros rMSSD, SD1, SS y el ratio S/PS en ningún protocolo ni toma $(p<.05)$.

El lactato presentó una elevación al final de los cuatro protocolos $(p<.01)$, pero en el isotónico e isométrico (Tabla 2 y Figura 3d) fueron menores las concentraciones en comparación con el aeróbico y anaeróbico $(p<$ .05). Por otro lado, a pesar del corto tiempo transcurrido entre las tomas de muestras sanguíneas, se presentaron diferencias en las concentraciones de CK entre la toma basal y final $(p<.01)$, sin embargo, no fue así entre los protocolos (Tabla 2 y Figura 3e).

Finalmente, observamos que altas concentraciones de lactato en la toma final del protocolo aeróbico se asoció con una baja SD1 en la toma ejercicio $(r=-0.66, p$ $<.05)$, al igual que en la toma recuperación $(\mathrm{r}=0.78$, $p<.05)$; asimismo, cuando se presentaron altas concentraciones de $\mathrm{CK}$ en la toma final del protocolo anaeróbico, el ratio S/PS se incrementó $(r=0.69, p<$

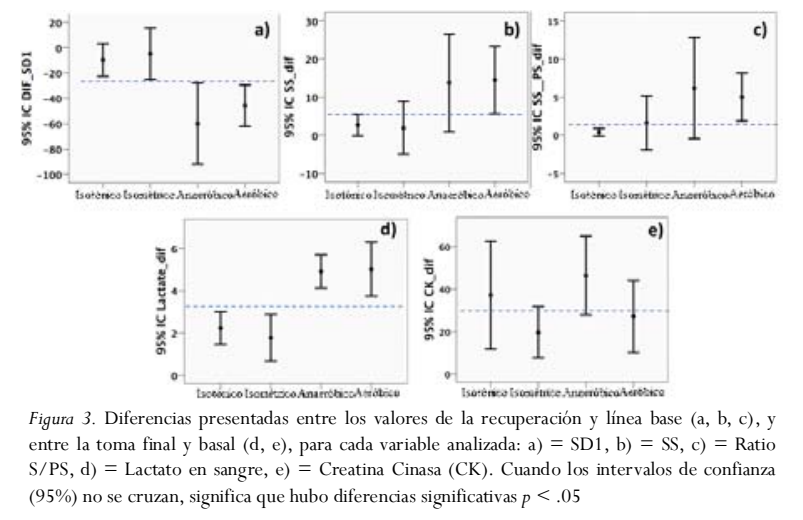

Tabla 2

Diferencias en el LA y la CK entre los protocolos de ejercicio.

\begin{tabular}{|c|c|c|c|c|c|}
\hline & a)Isotónico & a)Isométrico & a)Anaeróbico & a)Aeróbico & $\eta^{2}$ Parcial \\
\hline \multicolumn{6}{|c|}{ Lactato $(\mathrm{mmol} / \mathrm{L})$} \\
\hline Basal & $2.4 \pm 1.1(1.7-3.1)$ & $2.5 \pm 1.1(1.7-3.2)$ & $2.7 \pm 0.7(2.2-3.2)$ & $2.7 \pm 1.2(1.8-3.5)$ & 0.01 \\
\hline Post-ejercicio & $4.6 \pm 1.6(3.5-5.8) *$ & $4.2 \pm 1.5(3.2-5.3)^{*}$ & $7.6 \pm 1.7(6.4-8.8)^{\mathrm{a}, \mathrm{b}, \mathrm{k}}$ & $7.7 \pm 2.0(6.2-9.1)^{\mathrm{a}, \mathrm{b}, *}, *$ & 0.49 \\
\hline \multicolumn{6}{|l|}{ CK (UI/L) } \\
\hline Basal & $63 \pm 36(38-89)$ & $68 \pm 43(37-99)$ & $63 \pm 31(40-86)$ & $49 \pm 18(36-62)$ & 0.04 \\
\hline Post-ejercicio & $100 \pm 52(63-138)^{*}$ & $88 \pm 38(61-115) *$ & $109 \pm 51(73-146) *$ & $77 \pm 33(53-100) *$ & 0.08 \\
\hline
\end{tabular}

.05); mientras que en la prueba anaeróbica, altos niveles de $\mathrm{VO}_{2}$ max de los sujetos correlacionaron con bajos niveles de concentración de $\mathrm{CK}$ en plasma $(\mathrm{r}=-0.72, p$ $<.05)$.

\section{Discusión}

El propósito del estudio fue analizar las diferencias y asociaciones entre marcadores fisiológicos de carga interna en función de cuatro diferentes tipos de ejercicio, observándose respuestas más agudas y diferentes entre cada uno. Los resultados reflejan que en altas intensidades de ejercicio disminuye la variable parasimpática SD1, así como el SS que refleja la actividad simpática incrementa ligeramente, sin llegar a una completa recuperación tras 10 minutos post-ejercicio comparado con los niveles basales. En sujetos altamente entrenados expuestos a intensidades por debajo del umbral anaeróbico, se ha observado la completa recuperación de los valores de VFC entre 10-15 minutos de haber terminado el ejercicio; en altas intensidades, una completa recuperación puede superar los 30 minutos (Seiler, Haugen \& Kuffel, 2007). En relación con los resultados de este estudio, otras investigaciones también han encontrado diferencias entre la SD1 y SD2 en diversos tipos de ejercicio (Leicht, Sinclair \& Spinks, 2008). Durante estados de reposo y relajación el sistema parasimpático es predominante, por el contrario, durante el ejercicio, ambos sistemas simpático y parasimpático interactúan en función del tipo, intensidad y volumen de ejercicio realizado (Sarmiento et al., 2013; Sumi, Suzuki, Matsubara, Ando \& Kobayashi, 2006).

Por otro lado, observamos que, ante ejercicios de alta intensidad y duración, se presenta una gran disminución en la actividad parasimpática (baja SD1, y altos valores de SS y S/PS), y un mayor tiempo de recuperación; efectos que han sido observados en atletas de resistencia aeróbica en protocolos de carrera (Kaikkonen, Hynynen, Mann, Rusko \& Nummela, 2010). Una disminución en la VFC, tanto en reposo como en ejercicio es un indicador de la alteración en la homeostasis, y ésta es asociada a la fatiga, estrés y riesgo de mortalidad, a la inversa, una alta variabilidad indica una buena adaptación física al ejercicio y un buen estado de salud (De la Cruz, López \& Naranjo, 2008; Thayer, Yamamoto \& Brosschot, 2010).

Con respecto a la fatiga periférica, el metabolismo anaeróbico aumenta en todos 
los protocolos de ejercicio, ya que el lactato se elevó por encima del umbral anaeróbico (Ghosh, 2004). Además, el metabolismo anaeróbico fue mayor en los protocolos anaeróbico y aeróbico vs. los isométricos e isotónicos $(p<.05)$. El lactato es un subproducto de la actividad anaeróbica de la enzima lactato deshidrogenasa, a partir del piruvato y el dinucleótido de nicotinamida adenina reducido $\left(\mathrm{NADH}+\mathrm{H}^{+}\right.$), ambos productos de la glucólisis. El lactato basal es $<2 \mathrm{mmol} / \mathrm{L}$, y durante el ejercicio intenso se incrementa sobre los $4 \mathrm{mmol} / \mathrm{L}$ (Ramos-Jimenez et al, 2008). No obstante, el aumento del lactato no es el único causante de la fatiga periférica, diversos marcadores bioquímicos han sido asociados a ésta, entre los que se encuentran el incremento en el fosfato inorgánico $\mathrm{y}^{+}$, así como disminución en el $\mathrm{pH}$, ATP/ADP y ratio $\mathrm{PCr} / \mathrm{Cr}$, además de la inactivación de enzimas y canales iónicos, entre otros. Por otro lado, la correlación inversa $(r=-0.72, p<.05)$ entre el lactato y la activación parasimpática (SD1) observada en nuestros resultados, apoya la confirmación de hallazgos previamente reportados sobre la relación entre ambas variables, lo que indica la existencia de medición del umbral anaeróbico a través de la VFC (Karapetian, Engels \& Gretebeck, 2008).

Por último, a pesar del corto periodo de recuperación post-ejercicio y las limitadas diferencias encontradas, las concentraciones de CK en plasma se incrementaron significativamente respeto a la toma basal, pero no se presentaron diferencias entre los protocolos indicando una respuesta aguda al ejercicio (Ascensao et al., 2008). La concentración de CK en sangre depende de la edad, género, raza, masa muscular, actividad física (tipo, tiempo, intensidad y volumen), e incluso las condiciones climatológicas (Baird, Graham, Baker \& Bickerstaff, 2012; Brancaccio, Maffulli \& Limongelli, 2007). Por lo anterior, cuando se expone el cuerpo humano a fatiga aguda, el comportamiento de la VFC, CK y lactato es modificado de manera particular. En deportistas, la aptitud física, tipo de ejercicio e intensidad son factores que determinan el comportamiento de estos biomarcadores (Naranjo et al., 2015; RamosJimenez et al., 2008).

La carga de entrenamiento produce estrés y fatiga, a lo que el SNA responde de manera inmediata, siendo estimulada en sus dos diferentes ramas: simpática y parasimpática. De aquí la importancia de la medición de laVFC, la cual es un método seguro, simple, de bajo costo y no invasivo para conocer los cambios en el SNA provocados por el entrenamiento y la fatiga; siendo además un instrumento muy funcional para el desarrollo de programas de entrenamiento bajo un enfoque más científico y preciso de las cargas de trabajo, así como del proceso de recuperación de los deportistas.

Este trabajo tiene algunas limitaciones, entre ellas: el corto tiempo transcurrido entre las diferentes evaluaciones (72 h), y el bajo número de participantes. En función del pequeño rango de edad entre los participantes del estudio, así como las características específicas que se exploraron, la posibilidad de extrapolar los resultados a toda la población de deportistas es reducida. También, a causa del diseño experimental, las variaciones entre los tiempos de ejercicio en los protocolos fueron muy diferentes, lo cual podría ser un sesgo en los resultados observados. Además, tres días de descanso pudieron no ser suficientes para eliminar los efectos de la fatiga generada por la alta intensidad de los protocolos de ejercicio; a pesar de ello, el diseño de bloques al azar elimina el posible efecto residual de los ejercicios anteriores.

\section{Conclusión}

Los resultados del presente estudio corroboran que la VFC, CK, y las concentraciones de lactato son marcadores sensibles para detectar el estado de fatiga; tanto al tipo, duración e intensidad del ejercicio, siendo la VFC un marcador de mayor acceso, no invasivo, de fácil uso y útil tanto para los atletas y entrenadores.

\section{Conflictos de interés}

Ninguno de los autores declara intereses financieros ni de otra índole. Los experimentos se realizaron bajo las leyes actuales del país donde se llevaron a cabo.

\section{Referencias}

American College of Sport Medicine. (2016). ACSM's Guidelines for Exercise Testing and Prescription, 10th ed. LippincottWilliams \& Wilkins: Philadelphia, PA, USA, pp. 81-86. ISBN-10: 14963-3907-X.

Ascensao, A., Rebelo, A., Oliveira, E., Marques, F., Pereira, L. \& Magalhaes, J. (2008). Biochemical impact of a soccer match analysis of oxidative stress and muscle damage markers throughout recovery. Clin. Biochem, 41 (10-11), 841-851. doi: 10.1016/j.clinbiochem.2008.04.008

Baird, M.F., Graham, S.M., Baker, J.S. \& Bickerstaff, G.F. (2012). Creatine-kinase-and exercise-related muscle damage implications for muscle performance and recovery. J. Nutr. Metab, 1-13. doi: 10.1155/2012/960363.

Brancaccio, P., Maffulli, N. \& Limongelli, F.M. (2007). Creatine kinase monitoring in sport medicine. Br. Med.Bull, 81(1), 209230. PMID: 17569697

Bredin, S. S., Gledhill, N., Jamnik, V. K. \& Warburton, D. E. 
(2013). PAR-Q+ and ePARmed-X+: new risk stratification and physical activity clearance strategy for physicians and patients alike. Canadian family physician, 59(3), 273-277.

Buchheit, M.; Voss, S.C.; Nybo, L.; Mohr, M. \& Racinais, S. (2011). Physiological and performance adaptations to an in-season soccer camp in the heat: associations with heart rate and heart rate variability. Scand.J.Med. Sci.Sports, 21(6), e477-485. doi: 10.1111/j.1600-0838.2011.01378.x.

Cortex. Operador's Manual Metalyser 3B. (2016). Operation, calibration, maintenance. version $M L 3 B$ 2.1. Available online: https: / /www.procarebv.nl/wp-content/uploads / 2016/11/Cortex-Metalyzer-3B_-Handleiding.pdf (Accessed on 31 October 2018).

De la Cruz, B., López, C. \& Naranjo, J. (2008). Analysis of heart rate variability at rest and during aerobic exercise: a study in healthy people and cardiac patients. Br. J. Sports Med, 42(9), 715-720. doi: 10.1136/bjsm.2007.043646

Erickson, K., Côté, J., \& Fraser-Thomas, J. (2007). Sport experiences, milestones, and educational activities associated with high-performance coaches' development. The sport psychologist, 21(3), 302-316. doi.org/10.1123/tsp.21.3.302.

Ghosh, A. K. (2004). Anaerobic threshold: its concept and role in endurance sport. Malays. J. Med. Sci, 11(1), 24-36. PMCID: PMC3438148.

Gladden, L.B. (2004). Lactate metabolism: a new paradigm for the third millennium. J. Physiol, 558(1), 5-30. doi: 10.1113/jphysiol.2003.058701.

Grassi, B., Rossiter, B. \& Zoladz, J. (2015). Skeletal muscle fatigue and decrases efficiency: two sides of the same coin? American college of sport medicine 42 (2), 75-83. doi: 0091$6331 / 4302 / 75$ Y83

Halson, S. L. (2014). Monitoring training load to understand fatigue in athletes. Sports medicine, 44(2), 139-147.

Halson, S. \& Jeukendrup, A. (2004). Does overtraining exist? An analysis of overreaching and overtraining research. Sport med 34 (14) 967-981

Kaikkonen, P., Hynynen, E., Mann, T., Rusko, H. \& Nummela, A. (2010). Can HRV be used to evaluate training load in constant load exercises? Eur. J.Appl. Physiol, 108(3), 435442. doi: 10.1007/s00421-009-1240-1.

Karapetian, G.K., Engels, H.J. \& Gretebeck, R. J. (2008). Use of heart rate variability to estimate LT and VT. Int. J. Sports Med, 29(08), 652-657. doi: 10.1055/s-2007-989423.

Leicht, A.S., Sinclair, W.H. \& Spinks, W.L. (2008). Effect of exercise mode on heart rate variability during steady state exercise. Eur. J. Appl. Physiol, 102(2), 195-204. doi: 10.1007/s00421-007-0574-9.

Lucini, D., Marchetti, I., Spataro, A., Malacarne, M., Benzi, M., Tamorri, S., ... \& Pagani, M. (2017). Heart rate variability to monitor performance in elite athletes: Criticalities and avoidable pitfalls. International journal of cardiology, 240, 307-312.

Medina, M. de la Cruz, B.; Garrido, A, Garrido, M. \& Naranjo,
J. (2012). Normal values of heart rate variability at rest in a young, healthy and active Mexican population. Health, 4(7), 377-385. http://dx.doi.org/10.4236/ health.2012.47060

Naranjo, J., de la Cruz, B., Sarabia, E., de Hoyo, M. \& Domínguez, S. (2015). Two new indexes for the assessment of autonomic balance in elite soccer players. Int. J. Sports Physiol.Perform, 10(4), 452-457. doi: 10.1123/ijspp.20140235

Nosaka, K. \& Clarkson, P. M. (1996). Variability in serum creatine kinase response after eccentric exercise of the elbow flexors. Int. J. Sports Med, 17(02), 120-127.

Ramos-Jimenez, A., Hernandez-Torres, R.P., Torres-Duran, P.V., Romero-Gonzalez, J., Mascher, D., Posadas-Romero, C. \& Juarez-Oropeza, M.A. (2008). The respiratory exchange ratio is associated with fitness indicators both in trained and untrained men: a possible application for people with reduced exercise tolerance. Clin. Med. Circ. Respir. Pulm. Med., 2, 1-9. PMCID: PMC2990231

Ramos-Jiménez,A.; Hernández-Torres, R.P.; Wall-Medrano, A.; Juarez-Oropeza, M.A. \& Vera-Elizalde, M. (2013). Blood lactate kinetics on passive and active recovery. Gazz. Med. Ital, 172(5), 342-350.

Reynoso-Sánchez, L. F., Hernández-Cruz, G., López-Walle, J. M., Rangel-Colmenero, B. R. Quezada-Chacón, J.T. \& Jaenes-Sánchez, J. C. (2016). Balance de estrés-recuperación en jugadores universitarios de voleibol durante una temporada. Retos: nuevas tendencias en educación física, deporte y recreación, (30), 193-197.

Sarmiento, S., García-Manso, J.M., Martín-González, J.M., Vaamonde, D., Calderón, J. \& Da Silva-Grigoletto, M.E. (2013). Heart rate variability during high-intensity exercise. J. Syst. Sci. Complex, 26(1), 104-116.

Seiler, S.; Haugen, O.; Kuffel, E. (2007). Autonomic recovery after exercise in trained athletes: intensity and duration effects. Med. Sci Sports Exerc, 39(8), 1366-1373. doi: 10.1249/mss.0b013e318060f17d

Sumi, K., Suzuki, S., Matsubara, M., Ando,Y. \& Kobayashi, F. (2006). Heart rate variability during high-intensity field exercise in female distance runners. Scand.J.Med. Sci.Sports, 16(5), 314-320. doi: $10.1111 / \mathrm{j} .1600$ 0838.2005.00492.x

Thayer, J.F., Yamamoto, S.S. \& Brosschot, J.F. (2010). The relationship of autonomic imbalance, heart rate variability and cardiovascular disease risk factors. Int.J. Cardiol, 141 (2), 122-131. doi: 10.1016/j.ijcard.2009.09.543

Vernillo, G.;Agnello, L.; Barbuti,A.; Di Meco, S.; Lombardi, G.; Merati, G. \& La Torre, A. (2015). Postexercise autonomic function after repeated-sprints training. Eur.J. Appl. Physiol, 115(11), 2445-2455. doi: 10.1007/s00421015-3226-5.

Zulfiqar, U., Jurivich, D.A., Gao, W. \& Singer, D.H. (2010). Relation of high heart rate variability to healthy longevity. Am. J. Cardiol, 105(8), 1181-1185. doi: 10.1016/ j.amjcard.2009.12.022. 